\title{
Caracterização das hospitalizações por causas externas em uma unidade de terapia intensiva pediátrica
}

\author{
Characterization of hospitalizations for external causes in a pediatric intensive care unit \\ Caracterización de las hospitalizaciones debidas a causas externas en una unidad de cuidados intensivos \\ pediátricos
}

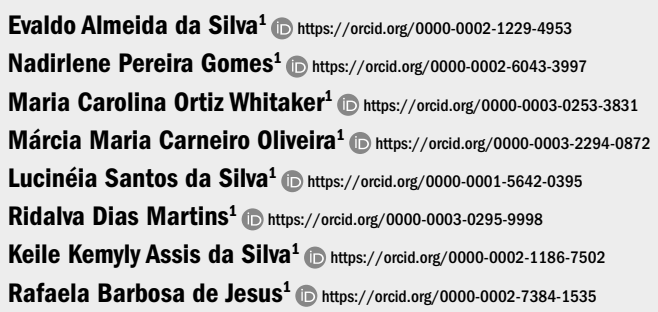

\section{Resumo}

Objetivo: Descrever os motivos de hospitalizações por causas externas em uma Unidade de Terapia Intensiva Pediátrica. Métodos: Trata-se de uma pesquisa descritiva, retrospectiva, desenvolvida em uma Unidade de Terapia Intensiva Pediátrica de um hospital público de referência em trauma na cidade de Salvador, Bahia. A coleta de dados efetuou-se por meio da consulta dos prontuários, norteada por um formulário de elaboração própria com informações de crianças e adolescentes hospitalizadas no ano de 2018 e 2019 . Foram avaliadas variáveis sociodemográficas (idade, sexo) e clínicas (motivo da internação: causas externas), por meio de análise descritiva das 305 internações infantojuvenis, que necessitaram de cuidados intensivos por causas externas.

Resultados: A maioria das hospitalizações ocorreu por queimaduras (24,3\%), quedas (22,6\%), acidentes de transporte $(22,3 \%)$ e violências $(11,5 \%)$.

Conclusão: Independentemente de sua classificação, as causas externas persistem acometendo crianças e adolescentes, sendo urgente a implementação de ações preventivas, que devem ser intensificadas em tempos de pandemia.

\section{Abstract}

Objective: Describe the reasons for hospitalizations for external causes in a Pediatric Intensive Care Unit.

Methods: This is a descriptive, retrospective research, developed in a Pediatric Intensive Care Unit of a public trauma reference hospital in the city of Salvador, Bahia. Data collection was carried out through the consultation of medical records, guided by a form of own elaboration with information from children and adolescents hospitalized in the year 2018 and 2019. Sociodemographic (age, gender) and clinical (reason for hospitalization: external causes) variables were evaluated by descriptive analysis of the 305 hospitalizations of children and adolescents who required intensive care due to external causes.

Results: Most hospitalizations occurred due to burns (24.3\%), falls (22.6\%), transport accidents $(22.3 \%)$ and violence $(11.5 \%)$.

Conclusion: Regardless of their classification, external causes persist, affecting children and adolescents, and it is urgent to implement preventive actions, which must be intensified in times of pandemic.

\section{Resumen}

Objetivo: Describir las razones de las hospitalizaciones debidas a causas externas en una Unidad de Cuidados Intensivos Pediátricos.

Métodos: Esta es una investigación descriptiva y retrospectiva desarrollada en una Unidad de Cuidados Intensivos Pediátricos de un hospital público de referencia de trauma en la ciudad de Salvador, Bahia. La recopilación de datos se llevó a cabo mediante la consulta de registros médicos, guiados por un formulario de elaboración adecuado con información niño y adolescentes hospitalizados en 2018 y 2019. Se evaluaron variables sociodemográficas (edad, sexo) y clínicas (motivo de hospitalización: causas externas) mediante un análisis descriptivo de las 305 hospitalizaciones de ninõs que requirieron cuidados intensivos para causa externas.

Resultados: La mayoría de las hospitalizaciones ocurrieron debido a quemaduras (24,3\%), caídas (22,6\%), accidentes de tránsporte $(22,3 \%)$ y violencia $(11,5 \%)$.

Conclusíon: Independientemente de su clasificación, persisten causas externas que afectando niños y adolescentes, y la aplicación de medidas preventivas es urgente, que debe intensificarse en tiempos de pandemia.

\section{Como citar:}

Silva EA, Gomes NP, Whitaker MC, Oliveira MM, Silva LS, Martins RD, et al. Caracterização das hospitalizações por causas externas em uma unidade de terapia intensiva pediátrica. Rev Soc Bras Enferm Ped. 2021;21(1):15-21.

\section{Descritores}

Unidades de terapia intensiva pediátrica; Crianças; Causas externas; Enfermagem pediátrica

\section{Keywords}

Pediatric intensive care units; Children; External causes; Pediatric nursing

\section{Descriptores}

Unidades de cuidado intensivo pediátrico; Niños; Causas externas; Enfermería pediátrica

\footnotetext{
${ }^{1}$ Universidade Federal da Bahia, Salvador, BA, Brasil.

Conflitos de interesse: nada a declarar.

Submetido: 11 de Novembro de 2020 | Aceito: 30 de Julho de 2021

Autor correspondente: Evaldo Almeida da Silva | E-mail: evaldo83@yahoo.com.br

Dol: http://dx.doi.org/10.31508/1676-3793202100003
} 


\section{Introdução}

As causas externas classificadas como eventos que podem ocasionar danos à saúde do indivíduo, podendo ocorrer de forma não intencional (acidente) ou intencional (violência), atualmente, apresentam-se como um dos principais motivos que contribuem para o elevado índice de morbimortalidade infantil. Essa preocupante realidade está atrelada a constante violação da garantia de direitos das crianças e adolescentes, que deveriam ser assegurados pela sociedade conforme é preconizado pela lei 8.069 (Estatuto da Criança e do Adolescente - ECA). Diante dessa situação, urgem estratégias de prevenção e enfrentamento desse agravo.

A mortalidade infantojuvenil por causas externas tem sido um problema de saúde pública no cenário mundial e nacional. Segundo a Organização Mundial de Saúde (OMS), em 2018, foram a óbito cerca de 6,2 milhões de crianças e adolescentes menores de 15 anos, sendo que mais 1,2 milhão mortes ocorreram por causas evitáveis. Ainda que nos últimos anos tenha havido redução nos números de mortalidade infantil no Brasil, as causas externas representam o segundo motivo de mortalidade em crianças e adolescentes. Os indicadores de mortalidade para a população infantojuvenil revelam as perdas de vidas precoces, todas as vítimas por causas evitáveis, onde se insere as violências. ${ }^{(1)}$

As causas externas, quando não ocasionam o óbito, podem provocar lesões que necessitem de tratamentos em serviços hospitalares, aumentando os índices de morbidade, nos casos mais graves com internações em Unidades de Terapia Intensiva. Estudo norte americano com objetivo de compreender a epidemiologia desses agravos identificou que aproximadamente 250.000 jovens entre 10 a 19 anos precisaram de atendimentos em serviços de urgência para tratamento por lesões decorrentes de causas externas. Nos hospitais deste mesmo país, ocorreram 7,7 milhões de internamentos por lesões em crianças e adolescentes. Uma pesquisa realizada na África, que avaliou durante o período de 11 anos os fatores preditores de hospitalização por queimaduras em crianças e adolescentes (0-16 anos), revelou extensas lesões corporais e situações de gravidade com evolução para paradas cardiorrespiratórias. No Brasil, as hospitalizações por causas externas em crianças e adolescentes durante os anos de 2017, 2018 e 2019 ocuparam o 5o lugar como motivos de hospitalização para essas faixas-etárias. ${ }^{(2-5)}$

Os dados de morbimortalidade refletem a violação de direitos e indicam os impactos negativos deixados pelos agravos por causas externas, que repercutem em prejuízos de ordem social e financeira. Considerando que vidas perdidas precocemente impactam no cenário econômico de todos os países e diante a necessidade de intervir neste contexto, no ano de 2018, a OMS lançou o World Health Statistics e o resultado desse relatório levou à criação da meta 3.6 dos Objetivos de Desenvolvimento Sustentável a qual indica que os países devem reduzir até o ano de 2030 a mortalidade e as lesões provocadas por causas externas. No Brasil, um exemplo desse compromisso para com o público infanto-juvenil remete ao Estatuto da Criança e do Adolescente (ECA) que assegura a proteção física, emocional e social e a punição mediante ação ou omissão que viole estes direitos fundamentais. No entanto, os números de crianças e adolescentes que sofrem causas externas, sejam elas acidentais ou provocadas, levam a reflexão acerca da interface destes eventos com a negligência e a violência, condutas também condenadas pelo ECA. ${ }^{(6,7)}$

Considerando que as crianças e adolescentes estão morrendo ou ficando com sequelas por questões relacionadas a acidentes e violências, é necessário que medidas de prevenção sejam revistas e ou implementadas com o objetivo de garantir à atenção integral a saúde de crianças e de adolescentes. Todavia, a fim de planejar medidas preventivas e protetoras, é preciso conhecer quais são os agravos mais prevalentes que levam a internações desse público. Nessa perspectiva, o estudo adota como objetivo descrever os motivos de hospitalizações por causas externas em uma Unidade de Terapia Intensiva Pediátrica.

\section{Métodos}

Esta foi uma pesquisa descritiva, retrospectiva, realizada em uma Unidade de Terapia Intensiva Pediátrica (UTIP) de um hospital público de referência em trauma na cidade de Salvador, Bahia, Brasil. No período de 2018 e 2019 foram hospitalizadas 443 crianças na unidade de terapia intensiva. Como critérios 
de inclusão foram avaliados prontuários de crianças e adolescentes vítimas de causas externas hospitalizadas no período de 2018 e 2019. Não participaram do estudo crianças/adolescentes hospitalizadas com outros diagnósticos. Das 443 internações que ocorreram entre os anos de 2018 e 2019, não participaram do estudo 138 prontuários por serem de crianças/adolescentes com outros tipos de diagnóstico. A amostra foi composta por 305 prontuários.

A coleta de dados foi realizada durante os meses de novembro de 2019 a março 2020. Foi efetuada por meio da consulta dos prontuários, norteada por um formulário de elaboração própria. As variáveis analisadas foram: sociodemográficas (idade, sexo) e clínicas (motivo da internação: causas externas). Realizada por uma equipe previamente treinada, os dados foram coletados, posteriormente revisados pelo pesquisador principal, sendo as inconsistências verificadas e corrigidas por pares.

Os dados coletados foram processados pelo Programa Estatístico Statistic Program for Social Sciences (SPSS) versão 25.0. Foram calculadas as frequências absolutas e percentuais para as variáveis categóricas, com a finalidade de identificar os tipos de causas externas que acometeram a amostra e caracterizá-las quanto ao sexo e a idade, sendo estes apresentados em formato de tabelas.

O estudo foi aprovado pelo Comitê de Ética em Pesquisa (CEP) da Escola de Enfermagem da Universidade Federal da Bahia (CAAE: 15413519.9.0000.5531) e pelo CEP da Secretaria de Saúde do Estado da Bahia, por meio da Escola Estadual de Saúde Pública Professor Francisco Peixoto de Magalhães Neto (CAAE: 15413519.9.3002.0052) que dispensou a aplicação do Termo de Consentimento Livre e Esclarecido, considerando que a fonte de dados retrospectivo em prontuários e o anonimato foi assegurado.

\section{Resultados}

Entre os anos de 2018 e 2019, foram avaliados 443 prontuários, dos quais 305 eram de crianças e adolescentes que necessitaram de cuidados em UTI devido às causas externas. A maioria das hospitalizações por este agravo deu-se por queimaduras $(24,3 \%)$, quedas $(22,6 \%)$, acidentes de transporte $(22,3 \%)$ e as violências $(11,5 \%)$.

Em relação a gravidade, foi utilizada a Escala de Coma de Glasgow na admissão das crianças e adolescentes na UTI, em que foi subdividida em leve (13-15 pontos) com 174 (57\%), moderada (9-12 pontos) com $20(6,6 \%)$, grave (3-8 pontos) com $91(29,8 \%)$ e não foram preenchidos os dados de 20 (6,6\%). O desfecho de alta em $286(93,8 \%)$, transferência para outro hospital em 3 (1\%) e óbito em 16 (5,2\%).

As principais causas externas encontradas nessa amostra foram destacadas em relação à faixa etária e sexo (Tabela 1). A faixa etária segue a categorização apresentada nos Sistemas de Informações do SUS (DATASUS), corroborando também com outros estudos.

Tabela 1. Distribuição de pacientes admitidos em uma UTIP por causas externas, de um Hospital Público de referência em trauma, quanto à faixa etária e sexo, nos anos de 2018 e 2019 (n=305)

\begin{tabular}{|c|c|c|c|c|c|c|}
\hline \multirow[t]{2}{*}{ Causas externas $\mathrm{n}(\%)$} & \multicolumn{4}{|c|}{$\begin{array}{l}\text { Faixa etária } \\
\text { Anos } n(\%)\end{array}$} & \multicolumn{2}{|c|}{$\begin{array}{l}\text { Sexo } \\
n(\%)\end{array}$} \\
\hline & $<1-(9,2 \%)$ & $1-4-(40 \%)$ & $5-9-(21,3 \%)$ & $\geq 10-(29,5 \%)$ & Masculino & Feminino \\
\hline Queimaduras $n=74(24,3)$ & $7(9,5)$ & $45(60,8)$ & $12(16,2)$ & $10(13,5)$ & $41(55,4)$ & $33(44,6)$ \\
\hline Queda $n=75(24,6)$ & $12(16)$ & $26(34,7)$ & $20(26,7)$ & $17(22,7)$ & $47(68,1)$ & $22(31,9)$ \\
\hline Ac. Transporte $n=68(22,3)$ & $1(1,5)$ & $16(23,5)$ & $20(29,4)$ & $31(45,6)$ & $51(75)$ & $17(25)$ \\
\hline Violência n=36 $(11,8)$ & $4(11,1)$ & $4(11,1)$ & $5(13,9)$ & $23(63,9)$ & $28(80)$ & $7(25)$ \\
\hline Bronco-aspiração n=22(7,1) & $4(18,2)$ & $15(68,2)$ & $2(9,1)$ & $1(4,5)$ & $12(54,5)$ & $10(45,5)$ \\
\hline Choque elétrico $n=01(0,3)$ & - & - & - & $1(100)$ & & \\
\hline Afogamento $n=9(3)$ & - & $7(77,8)$ & $1(11,1)$ & $1(11,1)$ & $4(44,4)$ & $5(55,6)$ \\
\hline Intoxicação Exógena n=8(2,6) & - & $5(62,5)$ & $1(12,5)$ & $2(25)$ & $4(50)$ & $4(50)$ \\
\hline Coice de animal $n=4(1,3)$ & - & $1(25)$ & $2(50)$ & $1(25)$ & $4(100)$ & - \\
\hline Ingestão de Corpo estranho $n=4(1,3)$ & - & $3(75)$ & - & $1(25)$ & $2(50)$ & $2(50)$ \\
\hline Lesão em academia $n=2(0,7)$ & - & - & - & $2(100)$ & $2(100)$ & - \\
\hline Exposição a forças mecânicas inanimadas* $n=2(0,7)$ & - & - & $2(100)$ & - & - & - \\
\hline
\end{tabular}

*Forças mecânicas inanimadas conforme CID 10 (W20- Impacto causado por objeto lançado, projetado ou em queda) que nessa pesquisa foram 2 quedas (muro e televisão) sobre a criança 


\section{Discussão}

O estudo revelou que queimadura, queda, acidente de transporte e violência constituem os principais motivos de hospitalizações por causas externas na UTIP estudada. Embora em ordem diferente, porém com percentuais semelhantes, exceto para as queimaduras. Pesquisa realizada no Rio Grande do Sul evidenciou serem estas as principais causas de internações hospitalares por causas externas: quedas (29\%), acidentes de transporte $(19 \%)$, as agressões $(9 \%)$ e as queimaduras $(8 \%)$. A diferença de percentual referente a queimadura, pode estar diretamente atrelada ao fato do lócus da pesquisa ser referência em trauma e dispor de um Centro de Tratamento de Queimados (CTQ) pediátrico. ${ }^{(8)}$

Estudo realizado em Campinas, São Paulo, ao qual foram observadas as características de crianças e adolescentes atendidas nos serviços sentinelas de urgência da cidade, mostrou que os acidentes mais recorrentes foram quedas, acidentes de transporte e queimaduras, sugerindo a ocorrência de diferenças, no que tange a classificação ordinal destas causas, segundo localizações geográficas e ou regionais. $\mathrm{O}$ acidente de transporte, a queda e a queimadura, respectivamente, também foram evidenciados enquanto motivos mais prevalentes de hospitalizações em Unidades de Terapia Intensiva Pediátrica (UTIPs) em pesquisa realizada durante sete anos na Austrália, com 542 casos de trauma pediátrico entre a faixa etária de zero a quinze anos. Destes pacientes, quase metade $(41,7 \%)$ estavam gravemente feridos, sendo necessária em alguns casos intervenção cirúrgica, o que sinaliza para os riscos que esses agravos podem ocasionar na vida dessas crianças. Apesar das diferenças na ordem relativas aos motivos que levaram a hospitalização, todas as pesquisas evidenciaram altos indicadores de internamentos por causas externas, com impacto significativo, causado por estes agravos, a vida desta população. ${ }^{(9,10)}$

Apesar do impacto que o internamento em UTIP pode gerar na vida das crianças /adolescentes/família, a maioria da nossa população chega na UTI com uma Escala de Coma de Glasgow entre 13 e 15 pontos, o que pode favorecer ao desfecho de alta da UTI em 93,8\%. A UTIP, principalmente a especializada, é um local que proporciona vantagens para uma boa recuperação e reduz a taxa de mortalidade. Trata-se de uma área com acesso restrito, sendo que as pessoas que circulam por esse local estão devidamente higienizadas e paramentadas, com o intuito de reduzir os riscos de contaminação para os pacientes. É importante destacar também que na UTIP existe uma equipe multiprofissional capacitada para prestar o melhor cuidado para crianças e/ou adolescentes que necessitam de cuidados intensivos, ou seja, que se encontram em situação clínica grave. ${ }^{(11)}$

Alerta-se ainda para a faixa etária entre 1 e 4 anos, responsável por $40 \%$ das hospitalizações. Nessa faixa etária, as crianças estão apresentando maior independência motora, não mais aceitando ficar somente nos braços, conduta que presume maior proteção atestada pelas menores taxas de quedas e queimaduras antes de completar 1 ano. Deste modo, a partir dessa idade, as crianças passam mais tempo ativas, porém ainda não apresentam coordenação motora plenamente amadurecida, realidade que as tornam mais vulneráveis aos acidentes, como as quedas. ${ }^{(12,13)}$

Outra característica própria do desenvolvimento das crianças, e que potencializa os riscos de acidentes, diz respeito à curiosidade, visto que tendem a explorar os diferentes espaços físicos, inclusive da própria casa, ficando assim mais propícias a acidentes de uma forma geral. Corroborando, estudo realizado no Hospital Geral de Maceió, Alagoas, aborda acerca da tendência das crianças em puxarem para si mesmas objetos que lhes chamam atenção, incluindo panelas e demais utensílios, o que pode provocar acidentes. Dentre as lesões nos pacientes pediátricos, a queimadura de $2^{\underline{ }}$ grau foi a mais predominante, sendo o tórax a região mais afetada, evidenciando dessa forma a gravidade que o ambiente doméstico, especialmente a cozinha, pode causar por ser um local onde ocorre o constante manuseio do fogo e preparo de comidas quentes. ${ }^{(14)}$

A pouca habilidade motora e a curiosidade, alinhadas a pouca ou nenhuma capacidade para prever e evitar situações de perigo acabam por predispor ao risco de acidentes. A realização de tarefas de acordo com sua fase de desenvolvimento influencia em seu processo de autonomia motora, fundamental na percepção de proteção e o senso do que é perigo. Com o amadurecimento motor e cognitivo, à medida que as crianças vão crescendo e se desenvolvendo, nota-se uma redução nas taxas de queimaduras e quedas.

Na contramão dessa tendência, se evidencia que os casos de acidentes de trânsito levaram as hospita- 
lizações em crianças maiores de 5 e 10 anos de idade. Isso pode estar relacionado ao fato de que crianças acima de cinco anos apresentam um maior controle motor para realizar algumas ações. Ratificando esse achado, estudo desenvolvido no Hospital Acadêmico Chris Hani Baragwanath (CHBAH), na África, aponta que as crianças na faixa etária entre 5 e 9 anos são as principais vítimas de acidentes de trânsito, sendo essa realidade atrelada ao início de uma maior independência. No continente africano, o fato de que muitas crianças durante essa idade estão começando a ir sozinhas para escola e outros lugares, aliado a falta de atenção no trânsito se constituem enquanto fatores de risco. Ciente dessa situação, em KwaZulu-Natal, cidade africana, foram implementadas medidas que proporcionaram um trânsito mais tranquilo nas áreas próximas das escolas, o que consequentemente diminuiu o índice de acidentes com crianças em $23 \%$. $^{(15)}$

Destaca-se que as diferenças encontradas nos diversos cenários mundiais podem estar relacionadas aos fatores sociais de cada local. Países desenvolvidos apresentam maior incidência por acidentes de trânsito, enquanto neste estudo encontramos a prevalência de queimaduras. Essa diferença revela que crianças estão em ambientes suscetíveis a queimaduras, como o domicílio, enquanto nos países desenvolvidos as crianças passam a maior parte do tempo nas escolas. O UNICEF (Fundo das Nações Unidas para a Infância) traz que as crianças nesses países possuem um maior acesso a educação que em países subdesenvolvidos, sobretudo nas séries iniciais dedicadas ao período da primeira infância. Infere-se que investimentos em educação, com o oferecimento de escolas públicas em tempo integral, podem favorecer para a minimização dos indicadores de acidentes domésticos e minimizar os gastos públicos com saúde que podem ser revertidos para a educação. ${ }^{(16)}$

Percebe-se ainda que, exceto os acidentes de transporte e a lesão em academia os demais motivos de internamentos por causas externas, ocorrem hegemonicamente no cenário domiciliar, com prevalência para o sexo masculino. Diversos estudos corroboram tais evidências, quando trazem que o domicílio é o lugar onde ocorre a maioria dos acidentes infantis, sendo este, um ambiente de grande risco para as crianças, com destaque para os meninos, uma vez que devido a construção de gênero eles são incentivados, desde pequenos, a realizarem atividades consideradas radicais e perigosas. Ainda que com uma maior prevalência em meninos, o presente estudo mostra que ambos os sexos estão vulneráveis a acidentes dentro de casa. Tal contexto de acidentes recorrentes pode ser justificado pelo fato dessas crianças e adolescentes permanecerem a maior parte do tempo em suas residências, onde acontece à maioria dos acidentes. ${ }^{(8,9,17)}$

Assim, independentemente do tipo de causas externas de internamento em crianças e adolescentes, merece destaque a preocupação com situações de violência, onde se insere a negligência, principalmente quando consideramos que mais de $60 \%$ dos casos vitimaram crianças $\geq 10$ anos. A este respeito, estudo realizado em um Hospital de Trauma na Dinamarca, durante 10 anos, evidenciou que os principais responsáveis pelas hospitalizações foram os acidentes e a violência, que se expressa não somente pela ação, como também omissão. Diante disso, é importante pontuar que a negligência representa uma das principais causas de violência infligida ao público infantojuvenil, responsável pela maioria das hospitalizações pediátricas por causas externas, causadas, dentre outros aspectos, pela omissão de cuidados dos pais e/ou responsáveis. Isso faz-se refletir que, embora seja apresentada em quarto lugar nos achados dessa pesquisa, a violência pode estar mascarada nas demais causas externas elencadas. ${ }^{(11,17)}$

Diante a vulnerabilidade de experienciarem acidentes/violências no espaço doméstico, urge uma atenção especial às crianças, sobretudo no contexto atual de pandemia pela Covid-19, devido o distanciamento social que resulta no confinamento das crianças no espaço doméstico. Soma-se o cenário comum de mães e pais acumulando tarefas domésticas e laborais, além de precisarem acompanhar as atividades escolares dos filhos, confinados meses em casa, sem lazer ou atividades em grupo. Toda essa situação é propícia, não só para a ocorrência de acidentes, como também para violência, principalmente quando associada à redução de carga horária ou mesmo perda do trabalho, que geram impactos sociais e econômicos nas vidas das famílias, situação que predispõe a violência. Assim, no período em que o distanciamento social revela-se enquanto medida efetiva para o controle da transmissão do vírus, a atenção para os acidentes e a violência no âmbito doméstico deve ser redobrada. ${ }^{(18)}$

Um alerta foi observado por meio de um estudo realizado em uma emergência pediátrica em Padova na Itália, o qual identificou que os internamentos por 
acidentes domésticos em crianças maiores de 1 ano, durante a pandemia pela Covid-19, aumentaram significativamente quando comparados com o mesmo período do ano anterior. ${ }^{(19)}$

Nesse interim, a Unidade de Terapia Intensiva Pediátrica (UTIP), se apresenta enquanto um importante mecanismo para promover a recuperação dos infantos internados. Haja vista as particularidades físicas, fisiológicas e emocionais desse público, uma Unidade de Terapia Intensiva, destinada ás crianças, permite melhor adequação do atendimento prestado, possibilitando uma recuperação mais rápida. ${ }^{(11)}$

No contexto preventivo para os acidentes e as violências, os profissionais da saúde e educação são de extrema importância no que tange ao processo de orientação aos familiares e/ou responsáveis, atuando de maneira incisiva quanto à necessidade do cuidado, da vigilância e da proteção para as crianças. Com isso, ressalta-se que as ações de prevenção desses agravos devem ocorrer nos diversos âmbitos do cuidado. Como instrumento para tal, dispõe-se da Estratégia de Saúde da Família (ESF) como um dos principais meios para a condução dessas ações preventivas, inclusive a partir de consultas das diversas especialidades do cuidado da população infantojuvenil. Esse espaço tem recebido destaque para a atuação das enfermeiras, não apenas pelo caráter educativo da formação, mas também por ocuparem cargos de gestão nos diversos espaços da Atenção Primária à Saúde. Nesse cenário, as profissionais, a partir da aderência ou não ao Programa Saúde na Escola (PSE), podem investigar as relações familiares para avaliação do grau de segurança e proteção das crianças e consequentemente mensuração do risco de acidentes por causas externas ou mesmo de negligências e/ou violências, sobretudo em tempos de distanciamento social quando suas redes de apoio social encontram-se limitadas, devido afastamento de pessoas confiáveis, como professoras, coleguinhas e familiares.

Infere-se que a mudança de conduta no cuidado ao público infantojuvenil requer ainda uma pactuação entre os espaços de atendimento e de formação profissional, a fim de contribuir para atuação em serviço de forma comprometida com a vida humana, principalmente quando se trata de seres com escassos recursos de defesa. Nesse sentido, experiência exitosa utilizada na investigação da violência defende o seguinte fluxo: identificar os casos, notificar, acolher, encaminhar a outros setores, orientações às famílias em situação de violência e atendimento/tratamento para as vítimas. ${ }^{(20)}$

O presente trabalho apresenta como limitação a visão unicêntrica do estudo e o seu lócus, que é uma unidade de referência em trauma. Dessa forma, acredita-se que seria importante o desenvolvimento de novos estudos de cunho multicêntricos randomizados com tamanho amostral maior.

\section{Conclusão}

O estudo revela que as queimaduras, as quedas, os acidentes de trânsito e a violência constituem os principais tipos de agravos que levam crianças e adolescentes a precisarem de um cuidado intensivo em unidade de terapia intensiva, evidenciando a prevalência de causas externas em uma amostra dessa população no estado da Bahia. Diante dessa caracterização, nota-se que, independentemente da classificação não necessariamente alinhada a outros estudos nacionais e internacionais, causas externas persistem acometendo crianças e adolescentes, sendo urgente a implementação de ações preventivas, que devem ser intensificadas em tempos de pandemia. Considerando a possível interface com situações de negligências/violências, urgem ações com foco no cuidado às crianças e adolescentes no âmbito das UTIP que investigue os casos de hospitalização por causas externas com foco no cuidado a este público e garantia de retorno a um lar que não as coloquem em risco para novos agravos. É preciso uma gestão que priorize a redução nos índices de morbimortalidade por causas evitáveis, seja através da institucionalização de protocolo para investigação de causas violentas para todos os casos de causas externas que gerem hospitalizações, seja por meio de medidas preventivas para prevenção e/ou reconhecimento precoce de situações de violência, o que pode ser favorecido a partir da Estratégia Saúde da Família, potencializada por meio da articulação com o Programa Saúde na Escola.

\section{Colaborações}

Silva EA, Gomes NP, Whitaker MCO, Oliveira MMC, Silva LS, Martins RD, Silva KKA e Jesus RB declaram que contribuíram com a concepção do projeto, análise 
e interpretação dos dados, redação do artigo, revisão crítica relevante do conteúdo intelectual e aprovação da versão final a ser publicada.

\section{Referências}

1. Organização Mundial de Saúde (OMS). Unicef e OMS dizem que taxas de mortalidade materno-infantil nunca foram tão baixas. Genebra: 0MS; 2020. [citado 2020 Abr 23]. Disponivel em: https://news.un.org/pt/story/2019/09/1687532

2. Ballesteros MF, Williams DD, Mack KA, Simon TR, Sleet DA. The Epidemiology of Unintentional and Violence-Related Injury Morbidity and Mortality among Children and Adolescents in the United States. Int J Environ Res Public Health. 2018;15(4):616.

3. Fomukong NH, Mefire AC, Beyiha G, Lawrence M, Edgar MM, Nkfusai NC, et al. Predictors of mortality of pediatric burn injury in the Douala General Hospital, Cameroon. Pan Afr Med J. 2019;33:189.

4. Safe Kids Worldwide (SKW). Why it matters. Washington: SKW; 2020. [cited 2020 Jun 23]. Available from: https://www.safekids.org/why-it-matters

5. Brasil. Ministério da Saúde. Sistema de Informacões sobre Mortalidade (Datasus/ Banco de Dados). Brasília (DF): Ministério da Saúde; 2018. [citado 2019 Set 25]. Disponível em: http://tabnet.datasus.gov.br/cgi/tabcgi.exe?sim/cnv/obt10uf.def

6. World Health Organization (WHO). World health statistics 2018: monitoring health for the SDGs, sustainable development goals. Genève: WHO; 2018. [cited 2020 Jun 24]. Available from: https://www.who.int/gho/publications/world_health_statistics/2018/en/

7. Brasil. Lei no 8.069, de 13 de julho de 1990. Dispõe sobre o Estatuto da Criança e do Adolescente e dá outras providências. Diário Oficial da República Federativa do Brasil, Brasília (DF); 1990

8. Bordin R,Abdala PR. Gestão em saúde no Rio Grande do Sul: casos, análises e práticas. 1a ed. Rio Grande do Sul: Pubblicato Editora; 2017.
9. Zimmermann SF, Fraga AM, Morcillo AM, Silveira NY, Antonio MA. Acidentes com crianças e adolescentes, segundo o Inquérito Sentinela. Rev Ciênc Med. 2018;27(3):115-124.

10. Coulthard MG, Varghese V, Harvey LP, Gillen TC, Kimble RM, Ware RS. A review of children with severe trauma admitted to pediatric intensive care in Queensland, Australia. PloS One. 2019;14(2):e211530.

11. Santomé LM, Leal SM, Mancia JR, Gomes AM. Crianças hospitalizadas por maus-tratos em UTI de serviço público de saúde. Rev Bras Enferm. 2018;71(3):1420-7.

12. Brito MA, Melo AM, Veras IC, Oliveira CM, Bezerra MA, Rocha SS. Fatores de risco no ambiente doméstico para quedas em crianças menores de cinco anos. Rev Gaucha Enferm. 2017;38(3):e2017-0001.

13. Konstantyner T, Mais LA, Taddei JA. Factors associated with avoidable hospitalisation of children younger than 2 years old: the 2006 Brazilian National Demographic Health Survey. Int J Equity Health. 2015;14:69.

14. Moraes MGL, Santos ML, Costa AB, Silva MR, Oliveira KCPN, Maciel MPGS. Causas de queimaduras em crianças atendidas em um hospital público de Alagoas. Rev Bras Queimaduras. 2018;17(1):43-49.

15. Patel N, Khofi-Phiri I, Mathiva LR, Grieve A, Loveland J, Nethathe GD. Trauma related admissions to the PICU at Chris Hani Baragwanath Academic Hospital, Johannesburg. Pediatr Surg Int. 2017;26(33):1013-101.

16. Fundo das Nações Unidas para a Infância (UNICEF). A World Ready to Learn: Prioritizing quality early childhood education. Nova York: UNICEF; 2019. [cited 2020 Jun 24]. Available from: https://data.unicef.org/resources/a-world-ready-to-learn-report/

17. Ekström DS, Larsen RH, Lauritsen JM, Færgemann C. Children and adolescents admitted to a university-level trauma centre in Denmark 2002-2011. Dan Med J. 2017;64(4):A5356.

18. Marques ES, Moraes CL, Hasselmann MH, Deslandes SF, Reichenheim ME. A violência contra mulheres, crianças e adolescentes em tempos de pandemia pela COVID-19: panorama, motivações e formas de enfrentamento. Cad Saude Publica. 2020;36(4):e00074420.

19. Bressan S, Gallo E, Tirelli F, Gregori D, Dalt L. Lockdown: more domestic accidents than COVID-19 in children. Arch Dis Child. 2021;106(2):e3.

20. Lourenço LM, Paula Junior GV. Violência Doméstica na Agenda da Saúde: Crenças de Gestores de uma Microrregião. Gerais Rev Interinst Psicol. 2020;13(1): e130112. 